\title{
Epistemologia contemporânea em ciências sociais: das questões gerais aos estudos da mediatização na comunicação ${ }^{1}$
}

\author{
Claudiane Carvalho' \\ https://orcid.org/0000-0002-5000-1074 \\ I - UFBA \\ Salvador (BA), Brasil
}

Resumo: A partir da reflexão sobre os aspectos constituintes da epistemologia contemporânea em ciências sociais, este artigo busca delinear perspectivas e desafios aos estudos da mediatização na comunicação. Por meio de uma revisão bibliográfica, apresentamos, inicialmente, um breve panorama histórico da constituição das ciências sociais, a fim de apontar especificidades da pesquisa social e da pesquisa em comunicação e desafios impostos pelo fenômeno da mediatização aos estudos comunicacionais. Nesse trajeto, o texto revela que o percurso de constituição histórica e institucionalização das ciências sociais sinaliza pistas para enfrentamento dos possíveis obstáculos à elaboração do conceito e, quiçá, de uma teoria da mediatização.

Palavras chave: epistemologia contemporânea; ciências sociais; comunicação; mediatização.

Abstract: Contemporary epistemology in social sciences: from general issues to the studies of mediatization in communication - From the reflection on the constituent aspects of contemporary epistemology in social sciences, this article seeks to delineate perspectives and challenges to the studies of mediatization in communication. Through a bibliographic review, we present, initially, a brief historical overview of the constitution of the social sciences, in order to point out the specificities of social research and communication research and challenges imposed by the phenomenon of mediatization to communicational studies. In this way, the text reveals that the course of historical constitution and institutionalization of the social sciences points out clues for facing possible obstacles to the elaboration of the concept and, perhaps, of a theory of mediatization.

Keywords: contemporary epistemology; social sciences; communication; mediatization.

1 Este texto foi originalmente apresentado no GP de Teorias da Comunicação do $42^{\circ}$ Congresso Brasileiro de Ciências da Comunicação, realizado em Belém - PA, 2019 e é aqui publicado com atualizações. 


\section{Introdução}

A modernidade complexifica e problematiza a comunicação, cujas condições de produção, circulação e reconhecimento, sustentadas em aparatos sociotecnológicos, promovem a reconfiguração de práticas, discursos e relações sociais. A realidade construída socialmente pela mediação dos meios de comunicação implica a remodelagem de nossas experiências cotidianas, formas distintas de ser e estar no mundo (FRANÇA, 2001; MARTINO, 2017). Essa tessitura do social acarretou o uso exacerbado do termo comunicação ao longo do século XX, período no qual os estudos do campo comunicacional começam a ser delineados num cenário marcado pela instabilidade da própria noção de ciência². Inconstância, aliás, em muito patrocinada pelas ciências sociais.

A concepção de ciência, desenhada na modernidade, sofre abalos em seus aspectos epistemológicos e metodológicos desde o século passado. Ciências da natureza e sociais declinam da noção clássica e buscam uma acepção menos racionalizante e menos mecanicista. Nesse contexto, o próprio desenvolvimento de investigações sobre temas sociais nos séculos XIX e XX contribuiu para essa desestabilização, uma vez que a natureza do objeto de estudo e dos problemas da pesquisa social criou duas situações que suscitaram pontos de ruptura com o paradigma hegemônico da ciência (SANTOS, 2006). Na primeira, as ciências sociais alinharam-se à proposta positivista e usurparam da pesquisa social o seu caráter específico. Na segunda situação, os estudos invocaram a subjetividade, enquanto condição intrínseca à investigação social. Assim, não puderam aceder à objetividade e foram exilados ao território do "não científico". Em ambas as situações, o desconforto estava instalado, promovendo reflexões epistemológicas e metodológicas.

A abordagem de cientificidade norteada pela desdogmatização possibilita à epistemologia contemporânea o tensionamento da oposição entre ciências sociais e ciências naturais, resguardando a ambas a unidade científica e a especificidade inerente aos seus respectivos objetos e problemas de investigação. Além disso, novos olhares são lançados à relação entre conhecimento científico e senso comum e à abordagem do viés. Essas reflexões não conduzem a um relativismo epistemológico como alternativa ao peso histórico da racionalização, do mecanicismo e do monismo metodológico. O conhecimento objetivo e a busca da verdade (ainda que esta seja perecível nas ciências) não são preteridos, mas abrigam-se nas constatações de que o conhecimento é aproximado e o objeto, construído.

Erguida em face dessa fragilização da concepção moderna de ciência, a epistemologia contemporânea se reflete metodologicamente nas ciências sociais (e, aqui, se encontra a comunicação ${ }^{3}$ ) numa interpelação não dicotômica entre pesquisas qualitativas e

2 De forma geral, a ciência é "descrita como o uso de métodos sistemáticos de investigação empírica, a análise de dados, o pensamento teórico e a avaliação lógica de argumentos com o intuito de promover um bloco de conhecimentos sobre um determinado tema" (GIDDENS; SUTTON, 2017, p.44)

3 Neste texto, partimos do pressuposto de que, na construção da cientificidade, a comunicação enfrenta, por sua natureza e legado, os problemas comuns às ciências sociais como um todo, embora sejam resguardadas suas peculiaridades. Para uma discussão mais detida sobre o campo comunicacional, o delineamento de seu objeto de estudo e problemáticas, sugerimos Martino $(2017,2007)$ 
quantitativas, as quais não se anexam conceitualmente a determinados métodos. A busca, porém, deve ser por zonas de interseção e membranas permeáveis à natureza do objeto e ao "espírito do problema" (BACHELARD, 1996). Afinal de contas, o método, em suas dimensões técnica e filosófica, tem grau e abrangência de médio alcance e não passa incólume às vicissitudes das suas condições de produção (KAPLAN, 1972).

No intuito de traçar conexões entre epistemologia contemporânea, métodos nas ciências sociais e desafios impostos pelo fenômeno da mediatização ${ }^{4}$ (LUNDBY, 2014; HJARVARD, 2014; VERÓN, 2013, 2014; COULDRY, HEPP, 2017), este texto divide-se em três partes: no primeiro momento, a discussão é situada a partir de um panorama histórico da constituição das ciências sociais; em seguida, serão apresentadas especificidades da pesquisa social e da pesquisa em comunicação; por fim, discorreremos sobre os desafios impostos pelo fenômeno da mediatização aos estudos comunicacionais.

\section{O desenvolvimento das ciências sociais}

Neste tópico, ao olharmos, em retrospectiva, alguns episódios do desenvolvimento epistemológico e institucional das ciências sociais, visamos alcançar o êxito de localizar historicamente a discussão sobre a epistemologia contemporânea.

As ciências sociais são engendradas "no mundo moderno e seu desenvolvimento se insere no contexto de um processo evolutivo de especialização e de autonomização do saber ocidental" (PIRES, 2014, p.46), ancorado na causalidade e na formulação de leis a partir das regularidades observadas, com o objetivo de previsão e regulação dos fenômenos (SANTOS, 2006).

Assim, as raízes das ciências sociais remetem ao século $\mathrm{XVI}$, quando um projeto denominado sciencia (do latim, saber, conhecimento) introduz uma distinção entre a ciência e os outros saberes, por meio da busca do conhecimento secular e sistemático da realidade. No século XVII, o filósofo René Descartes, ao atrelar a lógica à matemática, defende a busca da verdade e a recusa de qualquer ideia que não passe por rigorosa investigação, evidenciando-se, pois, como verdadeira. Nessa proposta, os problemas precisam ser divididos em quantas partes forem necessárias e possíveis, e a resolução deve começar do mais simples ao mais complexo (DESCARTES, 2002). Os princípios cartesianos, associados ao empirismo de Francis Bacon e ao positivismo de Auguste Comte, deram o tom do fazer científico ao longo do século XIX.

Os estudos do mundo físico apropriaram-se, em primeira instância, da donominação ciência, marcando-a pela oposição natureza x homem, objeto x sujeito, científico x senso comum, entre outros. As chamadas ciências da natureza empreendem a busca pela verdade, a partir da distinção, traçada por Descartes, entre condições iniciais e leis da natureza, sendo as primeiras complicadas, das quais é necessário eleger o fato a ser observado,

4 Adotamos o termo mediatização em referência à noção de médium, à mediação inerente ao processo social de construção do sentido e à exposição dos processos de significação em dispositivos materiais. 
e as segundas são simples e comportam a regularidade, a medição, a classificação. Sobre essa diferenciação se assentam o pressuposto epistemológico e as regras metodológicas que asseveram a natureza do saber científico no paradigma moderno (SANTOS, 2006).

Nesse sentido, busca-se um conhecimento objetivo da realidade, com base em descobertas empíricas, rejeição do conhecimento dito a priori e de todas as pré-noções em sentido amplo. Vale assinalar que, no primeiro momento, essa distinção não é hierárquica, nem pejorativa, mas ela foi construída pelas ciências da natureza, que forjaram para si mesmas o título de modelo ideal de ciência (PIRES, 2014; SANTOS, 2006; ALFONSOGOLDFARB, 2004). De um lado, por conseguinte, estavam as ciências da natureza e, do outro, um campo, então, não científico, designado de forma vaga por filosofia, artes, humanidades, letras, belas artes etc.

As ciências sociais nascem no rastro de uma segunda distinção. "Elas aparecem de modo mais hesitante, sobretudo no interior desse domínio que se denominou de 'filosofia' ou 'letras' [...] Esse novo saber social procura, por sua vez, distinguir-se dos saberes filosófico e religioso" (PIRES, 2014, p. 46-47), ratificando, então, uma cisão abissal entre o científico e não científico (SANTOS, 2009).

No século XVIII, já é possível vislumbrar a "emergência de um saber social associado a uma exigência metodológica" (PIRES, 2014, p.47). Advindas desse processo, as ciências sociais surgem no século XIX, desenhando uma aproximação epistemológica e metodológica com as ciências naturais, ou seja, com a descoberta da realidade objetiva, por meio de métodos que exteriorizavam o objeto.

Nessa conjuntura, afastadas da filosofia e das letras e contíguas às ciências naturais, as ciências sociais subsistiam, internamente, com um dilema: como construir um conhecimento objetivo, submetido às verificações e refutações? No século XX, os positivistas defendiam o monismo metodológico. Nessa direção, o precursor da sociologia, Émile Durkheim, por exemplo, abordava o fato social enquanto coisa, demarcando sua exterioridade ao indivíduo e poder de coerção: "Comte, é certo, proclamou que os fenômenos sociais são fatos naturais, submetidos a leis naturais" (DURKHEIM, 2002, p.45). Aqui, generalidade e objetividade são reconhecidas como atributos do fato social, que pode ser investigado sob os auspícios do positivismo, numa abordagem estruturalista.

Na dimensão epistemológica, as contendas derivadas dessa postura científica culminam em discussões sobre que estratégias de conhecimento adotar. Que posição, ponto de vista ou atitude o pesquisador deveria assumir para produzir um conhecimento objetivo ou verdadeiro dos fatos sociais? A exteriorização do objeto deixa de ser a única alternativa e revela-se frágil e inconsistente.

Atinente à dimensão metodológica, o debate residiu na natureza dos dados e agrupou duas visões. A primeira, em sintonia com as ciências da natureza, prima, entre outras coisas, pelos números e busca uma unicidade na forma de tratamento dos dados, o quantitativo. 
Nesta vertente, os objetivos são de predição, regulação e precisão concedidas pelos números. A segunda, por sua vez, privilegia os dados qualitativos (PIRES, 2014). Nesta abordagem, a noção metodológica de precisão ganha outros contornos para forjar-se na descrição, afluindo à compreensão. No prolongamento dessas duas visões iniciais sobre a natureza dos dados, encontra-se a raiz das oposições entre o qualitativo e o quantitativo, uma dicotomia que parece não se afirmar diante dos aspectos do objeto, problema e delineamento do processo de pesquisa.

As ciências sociais nascem da perplexidade diante do mundo moderno, e os métodos construídos ao longo dos séculos XIX e XX tentam dar conta dos problemas dessa sociedade revolucionada pela industrialização, crises político-sociais, aparecimento do mercado, desmembramento entre os poderes do Estado e do clero, explosão demográfica, emergência do indivíduo moderno, entre outros (MARTINO, 2017). Nessas circunstâncias, é configurado um outro lugar para a comunicação no mundo social:

A comunicação somente ganha visibilidade, enquanto problema colocado à tradição do pensamento Ocidental, quando ela passa a ter o sentido de uma estratégia racional de inserção do indivíduo na coletividade, fenômeno correlato à emergência de uma forma de organização coletiva, cuja dinâmica não se assenta sobre os valores da tradição, mas sobre o consumo do presente. (MARTINO, 2017, p. 137)

Martino (2017) demonstra que a comunicação, além de constituir o fundamento da consciência humana, quer em sua forma coletiva ou individual, passa também a configurar uma estratégia racional de inserção do indivíduo na coletividade. O estudo da comunicação, portanto, ancora-se em sua dupla necessidade: 1) fundamento da condição humana e 2) vetor da instituição de relações, práticas e discursividades em um contexto de transformações sociais.

\section{Cientificidade nas ciências sociais e os estudos em comunicação}

A busca pela cientificidade e os dilemas que envolvem o tema são intrínsecos ao processo de instituição das ciências sociais. Vale destacar que a cientificidade pressupõe uma unidade no que tange ao científico sem que se oblitere as especificidades de cada "território" do saber (KAPLAN, 1972). Para tanto, as discussões sobre a natureza do objeto de estudo, o teor das descobertas, as posições e disposições para a busca da verdade e a relação com o senso comum animam o campo na defesa do seu capital simbólico (BOURDIEU, 1999).

A indagação sobre a cientificidade nos estudos sociais, considerada ultrapassada ou obsoleta, ainda anima discussões e desdobra-se em outra interrogação: como objetivar uma realidade da qual somos agentes? (MINAYO, GOMES, 2009; GIDDENS, SOUTTON, 2017).

A confirmação da vulnerabilidade das dicotomias que afiançaram a acepção de ciência tecida na modernidade evidencia o conhecimento como construído e aproximado. 
O conhecimento, que advém da relação em continuum entre sujeito e objeto, ocorre em determinadas condições de produção, circulação e recepção e, portanto, não pode ser reduzido à mera produção de significantes; é preciso levar em consideração sua dimensão sócio- histórica, sua natureza de uma produção irremediavelmente vinculada às condições antropológicas e sociológicas (FOUCAULT, 2004).

Essas condições de produção integram o contexto do fazer científico como um todo, cujo processo conjuga, simultaneamente, aquisição de um saber, aperfeiçoamento de uma metodologia e elaboração de normas e regras (BRUYNE et al, 1995). Esse processo só confere unidade à cientificidade porque ocorre nas tramas do que há de específico nas ciências.

Para Kaplan, o que singulariza as ciências sociais (chamadas por ele de ciências do comportamento, fazendo referência também às ciências humanas) é o seu objeto; as técnicas que o objeto admite ou requer são apenas secundárias. Um discriminante ímpar nesse objeto é o uso de signos: a constatação do homem como ser simbólico, cuja relação com o mundo, com o outro e consigo mesmo é mediada pelas linguagens. Assim, as ciências sociais examinam os processos em que a produção de significação desempenha um papel essencial.

Como a produção de sentido envolve a articulação espaço-tempo e é ancorada historicamente, Minayo (2009) destaca que o objeto de estudo é histórico, ou seja, as relações e práticas discursivas que constituem a sociedade são espaço-temporais. Por isso, para tais ciências, os dados "não são movimentos puros, mas ações - ou seja, atos praticados dentro de certa perspectiva que Ihes dá significação e objetivo" (KAPLAN, 1972, p.34).

O termo ação, como acentua Ricoeur (2010), em sentido estrito, remete ao fato de que aquilo que alguém faz implica objetivos, motivos; não se refere, portanto, ao movimento físico, mas guarda uma intencionalidade e provoca consequências. Segundo o filósofo, as ações são forjadas por aspectos de inteligibilidade, simbólicos e temporais. Assim, reconhecer o agente da ação e suas motivações - "quem" e "para quê" - está no âmbito da inteligibilidade. As ações são também históricas, localizadas no espaço-tempo, mediadas pela cultura.

Expandindo o raciocínio, considera-se que o objeto de toda pesquisa é construído, a despeito dos vínculos e menções ao "construtivismo" (PIRES, 2014). Compreende-se que a observação não é passiva, mas um processo de semiose, marcado pela nossa inscrição histórica e cultural no mundo. Em outros termos, a ciência não se inicia com um olhar distanciado e neutro dos fatos, mas com uma visão teórica que organiza nossa observação.

No mais, Berger e Luckmann (2008) apontaram outros ângulos para os estudos em ciências sociais ao demonstrarem que a realidade é uma construção social, tecida nos processos de socialização constituídos e constitutivos da linguagem. Estas duas condições - 1) o objeto científico é construído e 2) a realidade social é produzida pelos seres humanos - são inerentes a qualquer processo de pesquisa social e não podem ser anexados ao construtivismo. 
Descartada a indexação equivocada entre a construção do objeto e o construtivismo, é preciso romper também com a suscetível vinculação entre objeto cientificamente construído e subjetivismo. Ligação que pode ser dissolvida por meio do entendimento de que entre o objeto de estudo e a realidade ocorre uma aproximação, e não uma correspondência. Afinal, o conhecimento que o pesquisador elabora é aproximado, ou seja, não abrange toda a realidade, os erros são factíveis, deformações são introduzidas, há dimensões esclarecidas e outras obscurecidas (PIRES, 2014).

No que tange ao objeto de estudo da comunicação e sua composição, França (2001) explica:

\begin{abstract}
[...] o objeto da comunicação não são os objetos "comunicativos" do mundo, mas uma forma de identificá-los, de falar deles - ou de construí-los conceitualmente. E aqui chegamos ao veio tocado por nossa indagação: quando se pergunta pelo objeto da comunicação, não nos referimos a objetos disponíveis no mundo, mas àqueles que a comunicação, enquanto conceito, constrói, aponta, deixa ver. Essa é a natureza de um "objeto de conhecimento": construções edificadas pelo próprio processo de conhecimento, a partir de sua ferramenta e do seu "estoque cognitivo" disponível (o conhecimento com o qual se conta para poder conhecer mais) (FRANÇA, 2001, p.42).
\end{abstract}

A percepção de que o objeto de estudo é um procedimento metodológico do autor, que o constrói técnica e teoricamente, constitui um dos primeiros obstáculos do campo científico da comunicação, assegura França (2001). Na visão de Martino (2017), o objeto de estudo está nas práticas que envolvem os meios de comunicação: nenhuma outra disciplina, além da comunicação, tem a liberdade para explorar as práticas advindas pela introdução dos media na organização social (Ibidem, p. 138).

\title{
Ciências sociais e senso comum
}

Em seus primeiros estudos, as ciências sociais trataram o senso comum como o que devia ser repelido, negado em prol da cientificidade. Ao longo do tempo, porém, o tema mostrou-se complexo e marcado por distintas matizes, dada a posição do olhar do cientista em relação ao objeto e a abordagem em relação ao viés. Nesse cenário, é válido rever a pertinência ou não da ideia de ruptura nessa passagem entre o senso comum e o saber científico.

Boaventura de Sousa Santos (2006), ao divisar o colapso do paradigma da ciência moderna e a emersão de um outro paradigma, pondera que uma visão renovada do processo científico esmaece a dicotomia entre o conhecimento científico e o ordinário, dessacralizando a ciência e reposicionando o senso comum.

Houle (1986) lembra que, no primeiro momento, a forma de conhecimento auferida pelo pesquisador das ciências sociais é o senso comum. Harding (1986), por sua vez, 
destaca a impureza inerente ao conhecimento científico e também ao senso, a qual não possibilita abarcar o objeto em completude, seja por um prisma ou pelo outro. Além disso, o senso comum instiga a pesquisa: "Início indispensável, passagem obrigatória, estadia forçada, fonte de criatividade e de correção de erros, processo de crítica semelhante e retorno necessário: desde então, qual o sentido pode ainda haver em continuar falando em ruptura?" (PIRES, 2017, p.9)

No mais, o conhecimento desenvolvido pela ciência tem origem no senso comum e retorna para ele, por meio da reflexividade. Sublinha-se ainda o duplo movimento entre ciência e prática, que envolve uma desnaturalização, processo que impulsionou e legitimou os estudos no campo da comunicação:

A modernidade transformou a comunicação em problema; levantou questões em torno de uma prática até então natural, naturalizada - prática esta que desde então se impôs aos homens como algo a ser melhor conhecido. A resposta ao desafio é a apreensão e a conformação desses estímulos na forma de um "objeto" recortado; é a transformação do problema sentido em problema formulado; é construir desse problema um objeto do conhecimento. (FRANÇA, 2001, p.46)

Faz-se crucial destacar que, mediante métodos e técnicas de pesquisa e uma prática disciplinada e disciplinadora, o conhecimento científico visa reposicionar o senso comum. Aqui, não se enfraquece o fato de que a ciência é um fenômeno social e histórico, passível de erros e à mercê das tramas ideológicas e do poder. Porém, conclama-se uma visada filosófica ao fazer científico, calcada na crítica de seus métodos e resultados e na validação de seus processos.

\section{As descobertas nas ciências sociais}

Em sua aula inaugural no Collège de France, em 1982, o sociólogo Pierre Bourdieu atentou à condição das ciências sociais de transformar em descoberta o que se tornou invisível por excesso de visibilidade.

A análise, exposta na obra Lições da Aula, indica que a primeira característica dessas descobertas científicas é esta: mostrar uma evidência de tal maneira que faz dela uma revelação. A segunda é que são indesejáveis e impopulares, mexem com as defesas coletivas ao ostentarem o que era para ficar escondido. O terceiro atributo diz respeito ao fato de que, por conta do peso que suscitam, essas descobertas podem ficar esquecidas. Além disso, com o tempo, também podem cair na banalidade, na trivialidade.

Nas ciências sociais, geralmente, o êxito e resultados das descobertas dependem das relações sociais, da ação de uma coletividade mais ampla. E, por fim, a aplicação dessas descobertas está subordinada a uma conjunção de interesses políticos e econômicos, pois, geralmente, elas provocam um abalo no status quo (PIRES, 2014). 


\section{A busca da verdade nas ciências sociais}

Como apreender a verdade sobre o mundo social? Esta indagação permeia a busca da objetivação nas ciências sociais. Trata-se de uma reflexão que tangencia tanto o plano epistemológico quanto o metodológico e faz entrever que a busca pela cientificidade se alinha à busca pelo(s) método(s).

As articulações metodológicas e epistemológicas geraram, numa leitura retrospectiva, três vertentes de pesquisas. Tendo como referência as ciências da natureza, a primeira vertente preza por dados quantitativos e exteriorização do fato social. A segunda, por sua vez, baseia-se numa oposição filosófica entre o mundo da natureza e o mundo da cultura. Essa pespectiva valoriza "o fato de que a subjetividade adquire uma importância capital para a compreensão, interpretação e explicação científica das condutas humanas" (PIRES, 2009, p.72). Aqui, ocorre um acoplamento entre pesquisas quantitativas e qualitativas.

O terceiro modelo de busca da verdade descende de uma leitura marxista do pensamento de Hegel, no âmbito sociológico. Aqui, o mérito do ponto de vista partidário é defendido pela antropologia diante das problemáticas advindas do etnocentrismo e do racismo. Nessa ótica, a abordagem qualitativa conquista um lugar privilegiado nas reflexões e desenhos de pesquisa, especialmente a partir dos anos 1970 .

Ao contemplar essas vertentes, é plausível cogitar as negociações e articulações entre elas. Se o fio de Ariadne no processo de pesquisa consiste em atentar-se aos aspectos do objeto e ao problema, as prováveis conexões devem considerar as pertinências e revisões necessárias face aos fenômenos contemporâneos, a exemplo da mediatização.

\section{Em nome do objeto e do problema de pesquisa e os estudos do fenômeno da mediatização}

Se as ciências sociais nascem da perplexidade diante do mundo moderno, na contemporaneidade, olha-se para a história dessas ciências a fim de construir aportes teórico-metodológicos que deem conta de uma sociedade mediatizada, revolucionada pela digitalização, internet e web, redes sociais, tecnologia móvel e datificação.

Numa sociedade mediatizada, a comunicação com/pelos media é contemplada como ambiente, que reconfigura, estruturalmente, as relações e práticas sociais. O fenômeno apresenta, entre outras, uma nova agenda de pesquisa para questões antigas, a saber: quais são as implicações dos media na constituição da sociedade e da cultura contemporâneas. Segundo Hjarvard (2014), a perspectiva teórica da mediatização difere dos paradigmas dos efeitos e da audiência, porque aborda uma sociedade mediatizada em detrimento da sociedade mediada.

O fenômeno, que congrega conceitos conflitantes, também alimenta a aspiração de se construir uma teoria que possibilite uma leitura do social a partir do papel e 
da influência dos meios de comunicação (LUNDBY, 2014). As investigações desenvolvidas sobre o tema, geralmente, são alocadas em três vertentes: institucional, construcionista e materialista/ tecnológica.

Pesquisador expoente na primeira vertente, Stig Hjarvard concebe um olhar abrangente para os meios, considerando-os como tecnologias, instituições e formas estéticas, os quais mudam as relações entre instituições e indivíduos, impactando, portanto, diferentes dimensões das interações sociais e, consequentemente, do mundo social.

Hjarvard distingue a mediatização como um fenômeno da modernidade tardia, que se intensificou, especialmente, nas últimas décadas do século XX com o surgimento dos meios digitais e a expansão acentuada da comunicação no tempo, no espaço e na modalidade (HJARVARD, 2014). Essa compreensão foi compartilhada pelo semioticista Eliseo Verón desde suas primeiras produções sobre o tema nos anos 1980 até início da década de 2010, quando lançou ao conceito uma interpretação semioantropológica, aportada na corrente construcionista.

Em suas últimas produções, Verón $(2013 ; 2014)$ aborda o conceito no contexto da evolução da espécie. Nesse horizonte, a mediatização consiste na sequência histórica de fenômenos mediáticos, ou seja, nos processos históricos de exteriorização dos processos cognitivos por meio dos dispositivos técnicos da comunicação. Nessa ótica, o fenômeno constitui o resultado operacional de uma dimensão nuclear da nossa espécie biológica, mais precisamente sua capacidade de semiose (VERÓN, 2014). O conceito de mediatização, para Verón, está ancorado nos usos e apropriações sociais dos dispositivos técnicos, que promovem autonomia e persistência dos discursos no espaço-tempo e a revolução do acesso.

Enquanto a perspectiva construcionista busca entender a influência dos meios de comunicação nos processos sociais de construção da realidade e produção do sentido num nível macro, a abordagem institucional permite fazer inferências e hipóteses sobre as relações microssociais de indivíduos de um determinado setor cultural ou social, sem intentar generalizações, mas contentando-se com abordagens de médio alcance (HJARVARD, 2014).

Apesar dos níveis e graus de abrangência diferenciados e especificidades epistemológicas, as três vertentes (inclui-se, aqui, a materialista, que tem foco na relação entre o humano, a técnica e a tecnologia) partilham a visão de que os momentos marcantes da história da mediatização não são lineares, mas complexos e contextualizados. Além disso, alteram o mundo social e suas relações e provocam a aceleração do tempo histórico (LUNDBY, 2014).

Essas três vertentes certificam a condição do objeto de estudo enquanto uma construção tributária dos percursos epistemológicos e metodológicos do cientista. Nenhum estudo vai dar conta do fenômeno na sua integralidade e, no intuito de minimizar as lacunas, autores como Andreas Hepp (2014), por exemplo, associam as abordagens 
construcionista e institucional em suas investigações. Hepp realça o primado do problema de pesquisa para definição do aporte teórico-metodológico, além de avultar o caráter interdisciplinar dos estudos.

Tendo em vista que a realidade social é uma produção humana simbólica, os desafios impostos pelas investigações sobre a mediatização condensam pontos e questões que são inerentes à trajetória histórica e de institucionalização dos estudos das ciências sociais. Além disso, o fenômeno constitui um objeto atravessador a diferentes disciplinas sob essa alcunha de social.

A pergunta, sempre à espreita, sobre "como objetivar uma realidade da qual somos agentes" retoma a cena e solicita, do percurso já trilhado, as respostas. O trajeto já percorrido, por sua vez, indica que a chave está nas fronteiras, nas membranas, alheia às dicotomias.

Para dar conta, por exemplo, dos estudos sobre a mediatização de campos sociais da política, da religião, da educação etc (HJARVARD, 2014; COULDRY, 2014; HEPP, 2014; LUNDBY, 2014) ou para analisar as mudanças nos processos sociais de semiose (VERÓN, 2014); ou debruçar-se sobre as relações homem-máquina na constituição de novas sociabilidades (LATOUR, 1994), é necessário, por exemplo, romper com a polarização entre os métodos e pensar em pesquisas qualitativas e quantitativas em articulação.

Nesse sentido, Kaplan (1972) alerta que não se deve levar em conta apenas a metodologia, enquanto uma lógica ${ }^{5}$ reconstruída, mas é preciso ter em mente que, em todo ato de pesquisa, o cientista instaura e emprega uma lógica - a lógica em uso. Assim, institui-se um processo dialético entre lógica em uso e lógica reconstruída, no qual o próprio fazer indica, em certa medida, as coordenadas do seu êxito. O ato científico é marcado pela intertextualidade, recorre-se a pesquisas, métodos e técnicas já testados, mas de uma forma peculiar, pois as escolhas pela pesquisa qualitativa e/ou quantitativa devem ser guiadas pelas particularidades do objeto e pela natureza do problema.

No que tange à dimensão epistemológica, Pires (2014) defende que medidas qualitativas e quantitativas, a despeito das suas diferenças, comungam as mesmas funções: neutralizam certos aspectos da subjetividade do pesquisador, algumas pré-respostas ao problema e criam espaço para fazer emergir uma ideia nova ao longo da atividade de pesquisa.

Diante da heterogeneidade dos estudos da mediatização, observa-se que, além de condensarem problemáticas inerentes à pesquisa nas ciências sociais de forma ampla, ainda promovem uma intensificação dessas inquietações no campo da comunicação. Para Ferreira (2016), os estudos sobre o fenômeno podem reverberar numa teoria do social mediocêntrica, em prejuízo para uma abordagem mediocentrada. Dessa forma, nas negociações entre as disciplinas sociais, a comunicação pode projetar-se como uma exportadora de conceitos (CALHOUN, 2012).

5 Kaplan defende que o objeto de estudo da lógica é a adequação do espírito do cientista ao problema. 
A ação social numa sociedade e numa cultura mediatizadas apresenta camadas de sentido que impelem a uma desnaturalização da ambiência comunicacional, para abarcar os tipos particulares de interação gestados pelo medium. Aqui, avista-se um terreno fértil para o labor científico, uma vez que as investigações acerca do fenômeno da mediatização convergem as três dimensões que foram apontadas por Wolf (2003) como representativas dos avanços, a partir da década de 1970, das pesquisas no campo da comunicação, a saber: 1) a articulação entre sociologia do conhecimento e meios de comunicação de massa; 2) o investimento mais expressivo nas pesquisas sobre os processos de produção, circulação e reconhecimento da informação; e 3) os estudos de longo prazo.

A vinculação entre a sociologia do conhecimento e os meios de conhecimento representa um entrelaçamento entre as tradições europeia (abordagem crítica) e americana (pesquisa administrativa). Tal entrosamento abeira-se, por exemplo, das definições de meios de comunicação de massa que sustentam os estudos da mediatização. Para Verón, os meios consistem em configurações de usos que se tornam institucionalizadas em um lugar e tempo particulares ao redor de um dispositivo de comunicação (VERÓN, 2014, p.16).

Um outro sintoma dos avanços nos estudos comunicacionais é o interesse nos discursos informativos, cujas investigações confluem, na atualidade, especialmente às modalidades de circulação, que provocam mutações nas condições de produção e recepção (FAUSTO NETO, 2018). Os diferentes usos da internet vêm alterando o acesso ao conhecimento e à cultura, a relação com o outro e o vínculo social com as instituições. No que tange à produção social do sentido, "os processos de circulação são o novo campo de batalha, e essa guerra apenas começou" (VERÓN, 2013, p.282).

Por fim, os estudos da mediatização também consistem em abordagens a longo prazo, que se atêm às dimensões estruturantes da sociedade e da cultura contemporâneas.

Em linhas gerais, para os desafios impostos pela abordagem científica do fenômeno da mediatização, algumas respostas ou, no mínimo, algumas pistas encontram-se no percurso histórico e de institucionalização das ciências sociais. Os aportes epistemológicos, teóricos e metodológicos estimulam negociações e aberturas dialógicas, sob os auspícios da objetivação. Assim, o pluralismo epistemológico não pode tender a um relativismo radical e a metodologia deve designar uma reflexão transteórica e transdisciplinar da prática da pesquisa, sem que isso dissipe a construção do objeto de pesquisa no campo comunicacional.

Claudiane Carvalho é doutora e mestre em Comunicação e Cultura Contemporâneas pelo PÓSCOM- Universidade Federal da Bahia (UFBA) e graduada em Jornalismo na FACOM-UFBA. No estágio pós-doutoral, dedicou-se aos estudos de abordagens teóricas da mediatização e circulação. É pesquisadora do Centro de Estudos e Pesquisa em Análise do Discurso e Mídia.

claudianecarvalho.29@gmail.com 


\section{Referências}

ALFONSO-GOLDFARB, A M. O que é história da ciência. São Paulo: Brasiliense, 2004.

BACHELARD, G. A formação do espírito científico: contribuição para uma psicanálise do conhecimento. Rio de Janeiro: Contraponto, 1996.

BERGER, P.; LUCKMANN, T. A construção social da realidade: tratado de sociologia do conhecimento. Petrópolis: Vozes, 2008.

BOURDIEU, P. Economia das Trocas Simbólicas. São Paulo: Editora Perspectiva, 1999.

Lições da aula: aula inaugural proferida no College de France em 23 de abril de 1982. São Paulo: Ática, 1982. (Volume 8 da Série Temas)

BRUYNE, P; HERMAN, J; SCHOUTHEETE, M. Dinâmica da pesquisa em Ciências Sociais. Rio de Janeiro: Editora Francisco Alves, 1995.

CALHOUN, C. Comunicação como Ciência Social ( e mais).Intercom - RBCC. São Paulo, v.35, n.01, p.277-310, 2012

COULDRY, N. Mediatization and the future of the field theory. In.: LUNDBY, Knut (ed.). Mediatization of communication (Handbooks of Communication Science), v. 21. Berlin/Boston, De Gruyter, 2014, p.227-248.

COULDRY, N.; HEPP, A. The Mediated Construction of Reality. Cambridge: Polity Press, 2017.

DESCARTES, R. Discurso do Método. Regras para a direção do Espírito (Texto integral). São Paulo, SP: Martin Claret, 2002.

DURKHEIM, E. As Regras do Método Sociológico (Texto integral). São Paulo, SP: Martin Claret, 2002.

FAUSTO NETO, A. Circulação: trajetos conceituais. Rizoma, Santa Cruz do Sul, v. 6, n. 2, p. 8-40, 2018.

FERREIRA, G. M. Estudos de Comunicação: da enunciação à mediatização. Intexto, Porto Alegre, UFRGS, n. 37, p. 101-117, 2016.

FOUCAULT, M. A ordem do discurso. 10. ed. São Paulo: Loyola, 2004.

FRANÇA, V. V. O objeto da comunicação/ A comunicação como objeto. In: HOHLFELDT, A.; MARTINO, L. C.; FRANÇA, V. (org.). Teorias da Comunicação: conceitos, escolas e tendências. Petrópolis: Vozes, 2001.

GIDDENS, A.; SUTTON, P. W. Conceitos essenciais da Sociologia. São Paulo: Editora UNESP, 2017. HARDING, S. The Sciense Question in Feminism. Ithaca: Cornell University Press, 1986.

HEPP, A. Cultures of mediatization. Cambridge: Polity Press, 2012.

. As configurações comunicativas de mundos midiatizados: pesquisa da midiatização na era da 'mediação de tudo'. MATRIZes, São Paulo, v.8, n. 1, p. 45-64, 2014.

HEPP, A.; HJARVARD, S; LUNDBY, K. Mediatization: theorizing the interplay between media, culture and society. Media, Culture \& Society, Roterdã, v. 37, n. 2, p. 1-11, 2015.

HJARVARD, S. A midiatização da cultura e da sociedade. São Leopoldo: Editora Unisinos, 2013.

Midiatização: conceituando a mudança cultural e social. MATRIZes. São Paulo, v.8, n. 1, p. $21-44,2014$.

HOULE, G. Histoire et récits de vie: la redécouverte obligée du sens comum. In: DESMARAIS, D; GRELL, P. (org). Les récits de vie: théorie, méthode et trajectories types. Montreal: Saint-Martin, 1986. 
KAPLAN, A. A conduta da pesquisa: metodologia para as Ciências do Comportamento. São Paulo: EDUSP, 1975.

LATOUR, B. Jamais fomos modernos: ensaio da antropologia simétrica. Rio de Janeiro: Editora 34, 1994.

LUNDBY, K. (ed.). Mediatization of communication (Handbooks of Communication Science), v. 21. Berlim/Boston, De Gruyter, 2014.

MARTINO, L. C. Escritos sobre Epistemologia da Comunicação. Porto Alegre: Sulina, 2017. . (org.). Teorias da Comunicação, muitas ou poucas? Cotia: Ateliê Editorial, 2007.

MINAYO, M. C. S.; DESLANDES, S. F.; GOMES, R. Pesquisa social: teoria, método e criatividade. Petrópolis: Vozes, 2009.

PIRES, Á. P. Sobre algumas questões epistemológicas de uma metodologia geral para as ciências sociais. In.: POUPART, Jean et al. A pesquisa Qualitativa: enfoques epistemológicos e metodológicos. 4. ed. Petrópolis: Vozes, 2014.

RICOEUR, P. Tempo e narrativa. A intriga e a narrativa histórica. Tomo I. São Paulo: Editora WMF Martins Fontes, 2010.

SANTOS, B. S. Um discurso sobre as ciências. São Paulo: Cortez, 2006.

. Más allá del pensamiento abismal: de las líneas globales a uma ecologia de saberes. In. OLIVÉ, L;SANTOS, B. S. et al. Pluralismo Epistemológico. La Paz: Muela del Diablo Editores, 2009.

VERÓN, E. La semiosis social, 2: ideas, momentos, interpretantes. Buenos Aires: Paidós, 2013.

. Teoria da midiatização: uma perspectiva semioantropológica e algumas de suas consequências. MATRIZes. São Paulo, v.8, n. 1, p. 13-19, 2014.

WEBER, M. A ciência como vocação. In: WEBER, Max. Ensaios de Sociologia. Rio de Janeiro: LTC, 1982, p.154-186.

WOLF, M. Teorias da comunicação de massa. São Paulo: Martins Fontes, 2003.

Texto recebido em 01/10/2019

e aprovado em 09/12/2019. 